\title{
L'application du principe ALARA : un premier bilan
}

\author{
J. LOCHARD*, G.A.M. WEBB**
}

(Manuscrit reçu le 30 août 1984)

\begin{abstract}
RÉSUMÉ
Quels enseignements peut-on tirer des travaux concernant l'application du principe ALARA ? Sur le plan des fondements théoriques, les études qui se sont développées ces dernières années reposent toutes sur les deux postulats énoncés par la CIPR, à savoir la linéarité sans seuil de la relation dose-effet et le principe de l'allocation "optimale" des ressources de protection. Par ailleurs, la plupart des travaux affirment le caractère multidimensionnel des problèmes de protection, ce qui conduit, au niveau des méthodes d'aide à la décision, à envisager l'élargissement de la méthode coût-bénéfice. En ce qui concerne la quantification du principe ALARA, les techniques d'évaluation des coûts de protection et des niveaux d'exposition des populations semblent désormais bien établies. Des divergences d'approche subsistent cependant encore parmi les auteurs quant à l'évaluation monétaire du coût du détriment, la prise en compte du temps, des événements probabilistes, et aussi des "autres facteurs" non directement relevant des coûts de protection et du détriment radiologique. La réflexion doit se poursuivre désormais sur la place que peuvent occuper les études ALARA dans les processus décisionnels concernant la protection tant à la conception que pendant l'exploitation des installations. Des efforts particuliers doivent être consentis au développement de bases de données adaptées et à l'élaboration de techniques d'aide à la décision capables d'intégrer la multidimensionnalité des facteurs intervenants dans les choix de protection.
\end{abstract}

\section{ABSTRACT}

What can we learn from practical ALARA studies ? On the methodological point of view the different works developed these last years rely upon the two basic ICRP assumptions, i.e. : the linearity of the dose-risk relationship and the optimal ressource allocation principle. Furthermore, most authors seem now to assume the multidimensionality of radiological protection problems. This leads to envisage for decision-aiding techniques the enlargement of the cost-benefit method. As for the quantification process of ALARA principle, assessment techniques for both protection costs and levels of exposures of the various groups at risk are well established. Some differences can be however pointed out between authors concerning the monetary valuation of the detriment cost as well as the way to cope with time, probabilistic events and "other factors" not directly linked to protection costs and radiological detriment. Further consideration must be given to the exact role ALARA studies have to play in the decision-making process concerning conception and operation of installations. Particular efforts have to be put on the development of adequate data bases and also decisionaiding techniques able to take into account the multidimensionnality of factors involved in practical protection.

* Centre d'étude sur l'évaluation de la protection dans le domaine nucléaire (CEPN), BP 48, 92260 Fontenay-aux-Roses, France.

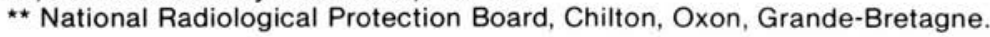




\section{AVANT-PROPOS}

L'article ci-après est une version française, entièrement révisée, d'une communication présentée sous le titre "ALARA in practice : how is it working ?" lors du $2^{\mathbf{e}}$ séminaire scientifique européen sur l'optimisation de la protection radiologique, qui s'est tenu à Luxembourg, les 8 et 9 novembre 1983. Cette communication que nous avons rédigée en tant que rapporteurs des deux groupes de travail qui s'étaient réunis préalablement les 26 et 27 avril 1983 afin de préparer le séminaire, avait pour objectif de synthétiser sur le plan méthodologique les principales conclusions des travaux menés aux cours de ces deux premières journées sur l'application du principe ALARA. Nous tenons tout d'abord à remercier la Commission des Communautés européennes, qui a bien voulu autoriser la publication de cette communication dans Radioprotection, malgré la parution officielle des actes du séminaire. L'originalité de ce dernier résidait dans le fait qu'il était presque exclusivement consacré à la présentation d'études de cas, à l'exception de trois communications préliminaires sur les aspects scientifiques et juridiques de l'optimisation (1). Ces études de cas réalisées par plusieurs équipes européennes avaient fait l'objet de discussions approfondies lors des journées préparatoires et l'article que nous vous présentons ici est une tentative de restitution des points marquants qui se sont dégagés de cette confrontation d'expériences très diverses. Quant au titre de cette communication, il appelle quelques commentaires que nous ferons sous forme de rappels concernant la signification du principe ALARA et son application dans le contexte spécifique français.

En termes généraux, le principe ALARA peut être défini ainsi : "toute activité médicale, scientifique ou industrielle susceptible d'entraîner une exposition de l'homme doit s'exercer dans des conditions de protection telles que les expositions aussi bien des travailleurs que du public soient maintenues au niveau le plus bas que l'on puisse raisonnablement atteindre, compte tenu des facteurs économiques et sociaux". Ce principe, souvent appelé principe d'optimisation de la radioprotection, ou encore principe d'évaluation de la radioprotection, est le deuxième principe du système de limitation des doses adopté en 1977 par la Commission internationale de protection radiologique (CIPR) dans sa publication 26 [5], le premier principe étant celui de la justification de l'activité, et le troisième celui de la limitation des doses individuelles. Ce système de limitation des doses a, depuis lors, été repris par la plupart des organismes internationaux concernés par la protection radiologique (AIEA, OMS, AEN (2)...) et figure dans la législation de nombreux pays. II a été également retenu comme fondement des directives les plus récentes de la Commission des Communautés européennes [8], ce qui explique la tenue du séminaire.

En ce qui concerne la France, le principe ALARA n'apparaît pas explicitement au niveau des textes officiels. Sur le plan pratique, cependant, l'idée de l'évaluation de la radioprotection en termes technico-économiques a fait lentement son chemin ces dernières années. De nombreux travaux méthodologiques, ainsi que des études de cas concrets ont été publiés notamment dans Radioprotection [19, 20, 22, 24, 25, 31] et les radioprotectionnistes qui ont touché de près à des exemples d'utilisation du principe ALARA reconnaissent l'intérêt que ce type de démarche présente comme outil d'aide à la décision au niveau des choix fondamentaux de radioprotection, tant à la conception que pendant l'exploitation des installations susceptibles d'entraîner l'exposition des travailleurs ou du public.

(1) Les lecteurs trouveront un bref compte rendu des études de cas présentées au cours de ce séminaire dans Radioprotection, 1984, n 1, p. 60.

(2) AIEA : Agence internationale de l'énergie atomique.

OMS: Organisation mondiale de la santé.

AEN : Agence pour l'énergie nucléaire. 
Il convient, enfin, de noter pour clore ces propos préliminaires que, sur le plan de la terminologie, l'expression en français "optimisation de la radioprotection" tend à être remplacée de préférence par les expressions "aide à la décision en matière de radioprotection", "gestion de la radioprotection" ou encore plus explicitement "évaluation technico-économique de la radioprotection". L'utilisation du concept d'optimisation soulève, en effet, plusieurs objections dans la mesure où il renvoie à un sens très restrictif en théorie économique qui ne correspond que très vaguement à la signification concrète du principe ALARA.

Nous espérons que cet article, malgré son caractère méthodologique, permettra aux lecteurs de se faire une idée plus concrète sur l'état actuel des applications du principe et indirectement aussi sur ses implications et ses véritables enjeux.

\section{INTRODUCTION}

L'histoire du développement du concept ALARA remonte maintenant à plus de deux décennies. Si l'idée que ce concept était quantifiable fit son apparition dans la terminologie CIPR dès la publication 22 [6], il a fallu, toutefois, attendre les principales recommandations de la publication 26 pour qu'elle se concrétise avec le rôle prépondérant accordé pour son application à l'analyse coût-bénéfice. En fait, la nécessité de quantifier le principe ALARA est toujours restée depuis lors un sujet de controverse, alors que ce ne fut jamais le cas pour le problème de la quantification des limites de doses. Pourtant, la CIPR spécifie clairement dans la même publication 26 que les valeurs proposées comme limites de dose reposent sur des jugements de valeur concernant les niveaux de risque associés à ces limites - niveaux de risque jugés "acceptables" par les experts de la Commission.

En ce qui concerne la mise en pratique du principe ALARA, cela n'a pas été une tâche des plus aisées. Très vite, des difficultés méthodologiques non pressenties par les promoteurs sont apparues; de plus, les bases de données dont disposaient ceux qui voulaient appliquer le principe étaient dans la plupart des cas totalement inadaptées. Quant au milieu des radioprotectionnistes, il était pour le moins sceptique quant à la nécessité de quantifier des éléments de décisions qui, jusqu'alors, semblaient relever du bon sens. Même si l'on peut admettre que certains praticiens de la radioprotection "faisaient de I'ALARA" sans le savoir, comme Monsieur Jourdain de la prose, le passage à la quantification fut cependant une étape déterminante. De ce point de vue, le second séminaire européen sur l'optimisation a largement fait la démonstration que l'étape avait été franchie avec succès.

Avant de passer en revue les principales questions soulevées par l'application du principe ALARA, il nous paraît important de rappeler les hypothèses fondamentales ainsi que le statut des techniques d'aide à la décision qui définissent le cadre théorique sur lequel il repose. II nous semble, en effet, que bon nombre de polémiques qui subsistent encore à propos de ce principe sont, à la base, alimentées par une mauvaise perception de ces éléments fondateurs qui ont été largement discutés et réaffirmés par les participants de la réunion de travail. 


\section{LES HYPOTHĖSES FONDAMENTALES DU PRINCIPE ALARA}

L'hypothèse vraiment primordiale, dont l'adoption conduit inévitablement au recours à un concept du type ALARA, est bien celle qui admet l'absence de seuil et la proportionalité entre le niveau de dose et la probabilité d'induction d'effets sanitaires. Cette hypothèse reste, cependant, pour de nombreux praticiens, non fondée. Pour clarifier le débat, il est à notre avis extrêmement important de bien faire la distinction entre, d'une part, le développement continu des recherches scientifiques qui ont pour objectif de faire la lumière sur l'action des rayonnements du point de vue physique et biologique, et d'autre part, la nécessité de recourir à un outil maniable sur lequel puisse reposer la pratique quotidienne de la radioprotection. Dans cette dernière optique, I'hypothèse de linéarité sans seuil remplit parfaitement son rôle et il devient de plus en plus évident qu'elle restera la seule hypothèse plausible pour la pratique, même si la non linéarité devenait une quasi-certitude. II apparaît, en effet, aux vues de plusieurs études récentes $[12,15]$, que les conséquences du choix de telle ou telle relation, comme l'a soulevé le comité BEIR [23], ne sont finalement pas aussi importantes du point de vue pratique qu'on aurait pu le craindre à première vue. Néanmoins, il faut bien garder à l'esprit qu'il ne s'agit que d'une hypothèse et qu'il convient donc de ne pas négliger les incertitudes qui lui sont inhérentes et qui viennent s'ajouter à celles qui caractérisent le système comme, par exemple : le caractère relatif ou absolu du risque, la non-prise en compte de l'âge et du sexe des personnes exposées, le recours à la dose moyenne à l'organe ou au tissu pour mesurer l'énergie absorbée, l'usage de coefficients de risque moyens ou encore l'usage de valeurs moyennes pour les paramètres métaboliques ou les habitudes alimentaires. Si de telles hypothèses ne sont pas très sensibles, en particulier lorsqu'il s'agit d'évaluer le risque pour de larges populations, il faut toutefois savoir relativiser les résultats numériques qui, même s'ils apparaissent précis, n'en intègrent pas moins des incertitudes qui, reconnaissons-le, sont rarement quantifiées ni même parfois évoquées dans les applications.

La seconde hypothèse fondamentale sur laquelle repose l'idée du principe ALARA est de nature économique. Enoncée en termes très généraux, elle consiste à admettre qu'en matière d'allocation des ressources de protection, il convient de rechercher un compromis entre les coûts engagés pour améliorer la protection, les niveaux de protection atteints et, éventuellement, d'autres facteurs socio-économiques lorsque ces derniers sont pertinents pour le système étudié. Cette idée est une transposition directe du principe de la détermination du niveau de contrôle optimal de la pollution, tel qu'il a été développé dans la théorie économique [21]. C'est le type de rationalité sur lequel repose généralement la justification des choix en matière de biens publics et la CIPR a cherché à le promouvoir dans le domaine de la protection radiologique.

L'hypothèse de linéarité de la relation dose-risque étant admise, la recherche d'un arbitrage entre les coûts de protection et les niveaux d'exposition résiduels apparaît donc comme une tentative de fonder le problème du choix de l'allocation des ressources de protection sur un critère socialement accepté. II s'agit là, en fait, d'une réponse à ceux qui prônent l'argument "du risque nul" et, ce faisant, oublient qu'au-delà d'un certain niveau de risque résiduel, toute amélioration peut aller à l'encontre 
d'une allocation rationnelle des ressources de protection, voire même à l'encontre de la protection de certains groupes soumis également au risque [22].

D'un point de vue pratique, la rationalité économique qui sous-tend le principe ALARA s'applique dans de nombreux domaines, et c'est pourquoi on peut trouver différents types de formalisation de ce principe selon qu'ils proviennent de l'économie ou de la recherche opérationnelle. Plusieurs techniques d'aide à la décision sont ainsi disponibles pour mettre en œuvre le principe ALARA et leur utilisation dépend essentiellement des caractéristiques du problème à traiter.

\section{LES TECHNIQUES D'AIDE A LA DÉCISION}

Tout décideur à qui il est demandé, lorsqu'il est face à un choix, s'il prend bien en compte l'ensemble des critères et des éléments pertinents qui caractérisent son problème, répond à coup sûr affirmativement. S'il lui est demandé comment, la réponse devient alors beaucoup plus évasive. En fait, les techniques d'aide à la décision ne sont rien d'autre que des outils pour répondre à cette seconde question et pour s'assurer, par conséquent, que la première est réellement satisfaite. Le recours aux méthodes d'aide à la décision ne conduit pas forcément à des choix différents de ceux qui auraient été fait intuitivement, mais il permet aux décideurs et également à tous ceux qui souhaitent comprendre les raisons sur lesquelles les choix sont fondés - d'identifier et d'évaluer les critères pertinents et, ce qui est beaucoup plus important, de rendre explicite le jeu des pondérations entre ces critères qui est inhérent à chaque décision. II s'agit là de rendre plus limpide le "comment avez-vous pris en compte ?".

Si l'on jette un regard en arrière, on peut évidemment beaucoup regretter que la CIPR ait, dès ses premières publications mentionnant explicitement le principe ALARA, mis l'accent avec autant d'insistance sur l'analyse coûtbénéfice. II en résulte que pour beaucoup de radioprotectionnistes il existe une stricte identité entre le principe et cette méthode. En fait, l'analyse coût-bénéfice n'est qu'une technique d'aide à la décision parmi d'autres, et de plus une technique qui soulève bien des objections dans la mesure où elle s'appuie sur des hypothèses assez restrictives qui en font une méthode souvent mal adaptée pour incorporer la complexité de nombreux problèmes de radioprotection. II faut néanmoins mentionner que la CIPR s'est légèrement rétractée sur le principe dans sa publication 37 [7], puisque quelques paragraphes, au niveau des considérations générales, ouvrent la possibilité du recours à d'autres méthodes, en particulier les méthodes dites multicritères. Les exemples d'évaluation présentés dans cette même publication 37 sont, cependant, tous traités dans le cadre d'une analyse coûtbénéfice des plus orthodoxes.

Pour toute méthode d'aide à la décision, on peut distinguer trois grandes étapes du point de vue de leur mise en œuvre : l'identification des actions possibles et des critères pertinents qui les caractérisent, l'évaluation quantitative de chacun des critères pour l'ensemble des actions et, finalement, la comparaison des actions selon les divers critères. Les méthodes les plus générales, comme les analyses de type multicritère, permettent 
d'appréhender tous les critères envisageables en les exprimant avec les unités dans lesquelles ils sont usuellement mesurés. Pour les techniques agrégatives comme l'analyse coût-bénéfice, seuls interviennent les critères qui peuvent de façon directe ou indirecte être exprimés en termes moné taires, les autres critères intervenant qualitativement extérieurement à l'analyse. Malgré ces différences apparemment irréductibles, il convient, cependant, de garder à l'esprit que chacune de ces techniques fait appel finalement aux mêmes types de jugements de valeur et d'hypothèses en ce qui concerne le délicat problème de la pondération des critères qui caractérisent les actions.

Certaines des études de cas présentées au cours du séminaire reconnaissent explicitement le caractère multidimensionnel des problèmes analysés, c'est-à-dire la nécessité de prendre en compte, au niveau des choix, d'autres critères que les seuls coûts de protection et les niveaux de risque résiduels associés à chacune des actions [17, 26, 30], mais, et cela n'est évidemment pas surprenant, la plupart des exemples traités s'appuient encore presque exclusivement sur des analyses de type coût-bénéfice.

\section{LA QUANTIFICATION DU PRINCIPE ALARA}

Même si les principes fondamentaux de l'évaluation technico-économique de la protection radiologique sont maintenant bien établis, la mise en œuvre de ces principes soulève néanmoins quelques difficultés, dont certaines n'ont pas encore trouvé de solutions vraiment satisfaisantes. Dans les paragraphes suivants, nous nous attacherons à souligner, pour les principaux aspects de la quantification du principe ALARA, les domaines pour lesquels un consensus semble désormais acquis et ceux qui restent problématiques.

\subsection{L'évaluation des coûts de protection}

La procédure d'évaluation des coûts de protection est, sans conteste, le domaine où un consensus s'est établi rapidement. Le cadre méthodologique pour prendre en compte à la fois les coûts d'investissement et les coûts d'exploitation est désormais bien fixé $[4,11,18,26,27]$, même si de légères différences subsistent en ce qui concerne l'utilisation des techniques d'actualisation.

Plusieurs études de cas ont, cependant, mis en lumière des difficultés au niveau de l'évaluation des coûts de protection, qui ne semblaient pas avoir jusqu'ici retenu l'attention des promoteurs du principe ALARA. La première concerne ce que nous appellerons le problème des interdépendances entre les coûts de protection et les coûts de production [11, 18]. En effet, dans de nombreux cas, il est tout à fait incorrect de ne considérer que l'accroissement des coûts de protection associés à la mise en œuvre d'une action de protection, car cette dernière peut également influencer positivement la productivité globale du système auquel elle s'applique. Dans ce cas, il convient donc de tenir compte de ces éléments positifs, qu'il s'agisse de bénéfices induits ou de coûts évités, en les retranchant des coûts de protection. Ce point, négligé jusqu'ici, mérite d'être souligné et systématiquement exploré dans la mesure où il démontre que faire de la protection n'est pas forcément contradictoire avec la recherche d'une meilleure productivité. 
La seconde difficulté est relative à la séparation des coûts de protection et des coûts de production. Dans de nombreuses situations, la distinction n'est pas facile à faire, et il est souvent nécessaire de décider de façon arbitraire la part du coût total d'un système qui peut être attribuée à la protection. Cet arbitrage relève, dans la plupart des cas, d'un jugement de valeur plus ou moins bien fondé et, par conséquent, une analyse de sensibilité devrait normalement accompagner ce type de démarche afin d'évaluer l'influence de telle ou telle hypothèse sur le résultat final.

\subsection{L'évaluation de l'exposition}

La difficulté majeure du point de vue de l'évaluation de l'exposition réside dans le fait que des mesures directes du niveau d'exposition des différents groupes de population soumis au risque sont, dans la plupart des cas, impraticables sinon impossibles. Comme l'évaluation de la performance des actions de protection requiert l'établissement d'une relation quantitative entre la source d'exposition et les doses individuelles et collectives qui en résultent, même si des mesures sont disponibles pour certaines situations existantes (c'est le cas pour les travailleurs, par exemple), il est toutefois nécessaire de déterminer cette relation pour les actions nouvelles envisagées. Cela ne peut être mené à bien qu'avec le recours aux techniques de modélisation. De nombreux modèles $[11,16,18,26,28]$, dont certains originaux, ont été présentés lors du séminaire, tant pour l'évaluation de l'exposition du public que des travailleurs. La modélisation apparaît ainsi comme une des dimensions essentielles de la mise en pratique du principe ALARA. Des progrès restent à faire dans ce domaine ; certains modèles devant encore être développés, d'autres améliorés, et il convient, là aussi, de ne pas négliger la prise en compte de l'incertitude inhérente aux techniques de simulation de la réalité.

\subsection{L'évaluation du coût monétaire du détriment radiologique}

L'analyse coût-bénéfice conduit, par définition, à exprimer les différents éléments qui entrent en ligne de compte au niveau de la décision en termes monétaires. Comme nous l'avons déjà souligné, l'importance accordée à cette méthode dans les premières recommandations de la CIPR a focalisé l'attention sur la fameuse question "du coût de l'alpha" (la valeur monétaire de l'unité de dose collective) puisque le modèle proposé repose sur l'hypothèse que le détriment radiologique est entièrement caractérisé par la dose collective (l'engagement d'équivalent de dose effectif collectif). En fait, il est apparu assez vite à ceux qui tentaient de passer aux applications que de nombreux praticiens de la radioprotection considéraient avec autant d'importance d'autres dimensions de l'exposition comme la distribution des doses individuelles, le fait qu'il s'agisse de doses délivrées à des travailleurs ou au public, ou encore le fait qu'il s'agisse de doses délivrées actuellement ou dans le futur. La CIPR pour répondre à ces préoccupations a introduit dans sa publication 37 [7] un second terme dit "terme bêta" qui n'a d'autre but que celui d'élargir le cadre initial de l'analyse coût-bénéfice afin d'y inclure ces dimensions particulières. 
Le seul système complet, présenté lors du séminaire, intégrant à la fois les termes "alpha" et "bêta", est celui qui a été développé au RoyaumeUni par le National radiological protection board (NRPB). Ce système est encore, à l'heure actuelle, en discussion mais l'introduction des deux termes permet de toute évidence de clarifier le débat sur le problème du choix des valeurs monétaires de l'unité de dose. II permet de comprendre, en particulier, les raisons pour lesquelles des valeurs très différentes de "alpha" ont pu être proposées ou même adoptées par des autorités. Certaines de ces valeurs, en effet, ne prennent en compte que le coût du détriment radiologique collectif, soit "alpha", alors que d'autres intègrent implicitement d'autres dimensions relevant de la composante "bêta".

De toute manière, la valorisation monétaire des dimensions intervenant au niveau du détriment radiologique reste une opération très délicate. Le recours à d'autres méthodes d'aide à la décision, comme les approches de type multicritère, ne supprime pas le problème, mais le déplace à un autre niveau. Au lieu de fixer des coûts monétaires sur les différentes composantes du détriment, il faut alors déterminer quel est le poids relatif de chacune de ces composantes, et cette tâche est tout aussi délicate que de fixer a priori des valeurs de "alpha" ou de "bêta". Aucune des communications n'a abordé de façon directe le problème de la fixation du coût monétaire du détriment, chaque auteur ou équipe se contentant de reprendre les valeurs déjà proposées dans la littérature. Cela est assez normal, puisqu'il s'agissait d'une réunion consacrée aux applications pratiques du principe ALARA, alors que la détermination du coût de l'unité de dose est une tâche qui relève des autorités nationales ou de certains organismes internationaux et non pas de ceux qui cherchent à mettre en œuvre le principe.

\subsection{La prise en compte du temps}

Le problème de la prise en compte du temps, en d'autres termes, le problème du poids à accorder aux doses qui seront reçues par les populations dans le futur, est pour certains l'une des questions à laquelle les techniques de mise en œuvre du principe ALARA doivent pouvoir répondre alors que pour d'autres il ne s'agit là que d'un faux problème. Aucune de ces deux positions n'est évidente en soi, et le problème apparaît incontournable ; une position doit être adoptée quant à la valeur à accorder aux doses qui seront délivrées dans quelques décennies ou même dans un futur lointain par rapport à celles délivrées dans l'immédiat ou dans un proche avenir. En fait, on peut voir dans cette question de l'étalement des doses dans le temps, la contrepartie de celle de l'étalement des coûts de protection et l'on sait que cette dernière est résolue par l'utilisation de la procédure d'actualisation. Le principe d'une pondération des doses dans le temps, réalisée soit par la technique de l'actualisation ou par le moyen de troncatures ou de coefficients atténuateurs, peut se justifier soit en fonction de principes relevant de l'éthique, en supposant, par exemple, que les générations futures bénéficieront du progrès technique et d'une richesse encore plus grande pour répondre au problème, soit, plus prosaïquement, en reconnaissant que l'incertitude concernant la prédiction des doses dans le futur est telle qu'elle enlève toute signification aux comparaisons d'options. 
Le problème de la prise en compte du temps dans la procédure de mise en œuvre du principe ALARA a naturellement été posé par les auteurs traitant des études de cas où des expositions du public à des radionucléides de longues périodes étaient en jeu [2, 4, 28]. Si l'ensemble des participants étaient d'accord pour admettre, comme le recommande la CIPR, qu'il faille minimiser le transfert de risque vers les générations futures, on a pu également constater un consensus sur le principe de l'atténuation de l'impact des doses résiduelles en fonction du temps. Cependant, parmi l'ensemble des techniques évoquées pour pratiquer cette atténuation, aucune n'a paru émerger comme préférable aux autres et il est certain qu'à terme une harmonisation des pratiques doit être trouvée à ce niveau pour rendre les comparaisons possibles.

\subsection{Le problème des probabilités}

Comment appliquer le principe ALARA lorsque les expositions dépendent d'événements probabilistes ? Cette question reste à l'heure actuelle non résolue bien qu'elle fasse l'objet de nombreuses discussions dans plusieurs instances internationales et qu'elle soit régulièrement débattue dans les réunions de praticiens. Plusieurs présentations au cours du séminaire ont abordé de manière directe le problème $[16,28]$. Si le concept d'espérance mathématique du détriment collectif apparaît chez les auteurs comme l'outil le mieux adapté pour comparer des événements de nature probabiliste ou non, la nécessité de distinguer différents type d'événements semble également s'imposer. C'est, en tout cas, ce qui est ressorti des discussions animées qui ont porté sur la question de l'intégration des événements probabilistes. Un certain consensus s'est dessiné pour admettre comme schéma minimal une distinction entre les événements rares (ceux que l'on pourrait définir comme ayant une probabilité d'occurence inférieure ou de l'ordre de $10^{-6}$ par an), les accidents (événements ayant une probabilité de l'ordre de $10^{-3}$ par an) et les incidents (événements pour lesquels des fréquences observées sont disponibles avec des probabilités supérieures à $10^{-2}$ par an).

\subsection{Les "autres facteurs"}

Au niveau de la prise de décision, il semble désormais acquis pour les décideurs qu'il n'est pas possible pour eux de s'en remettre purement et simplement aux seuls résultats des analyses quantitatives préconisées par le principe ALARA, d'autant plus s'il s'agit d'analyses aussi simplificatrices que les approches de type coût-bénéfice. II est clair alors que le rôle des "autres facteurs", c'est-à-dire les dimensions du problème qui ne relèvent ni des coûts de production, ni du détriment radiologique, doivent aussi intervenir dans la décision. La question de savoir comment ces autres facteurs peuvent être isolés et éventuellement quantifiés est, cependant, beaucoup moins évidente. Parmi ces facteurs, il faut certainement inclure les aspects techniques de la protection, comme la fiabilité des systèmes ou leur degré de développement technologique, qui sont difficiles à évaluer. II existe aussi beaucoup d'autres facteurs non techniques comme l'acceptabilité par le public ou par les travailleurs des niveaux de risques résiduels, ou encore des contraintes politiques que les décideurs ne peuvent absolument pas négliger. Bien que de nombreuses communications faisaient allusion à ces divers facteurs, et c'est là un progrès par rapport aux 
premiers travaux sur le principe ALARA, très peu cependant ont mentionné explicitement leur nature exacte. Parmi ces derniers travaux $[17,30]$ aucun ne franchit la barrière de la quantification. On peut espérer qu'avec le développement dans l'avenir des analyses de type multicritère, un tel obstacle pourra être surmonté.

\section{LES PROCHAINES ÉTAPES}

Les paragraphes suivants reprennent, sous un éclairage particulier, un certain nombre de points brièvement évoqués jusqu'ici, qui nous apparaissent, à la suite des discussions entre les participants, comme devant susciter dans l'avenir un travail de réflexion, afin d'affirmer encore davantage le caractère opérationnel du principe ALARA.

\subsection{L'intégration du principe ALARA dans les processus décisionnels}

Cette question a rarement été abordée dans la littérature jusqu'à présent et il faut reconnaître que la CIPR reste assez discrète sur la façon dont les principes qu'elle recommande peuvent s'insérer dans les processus décisionnels concernant la fixation des niveaux de protection des populations. II s'agit là, en fait, d'une attitude prudente face à l'extrême diversité des situations qui interdit d'envisager des solutions à caractère universel. $\mathrm{Si}$, au cours de ces journées de travail, on a pu noter une réelle convergence de vue parmi les participants sur les questions relatives à la rationalité et aux fondements théoriques du principe ALARA, ainsi que sur les procédures de quantification, par contre, en ce qui concerne l'utilisation de ce principe dans les processus décisionnels, des divergences marquées sont apparues. En simplifiant, on pourrait dire que pour certains l'application du principe ALARA apporte quelques renseignements intéressants sur les niveaux de protection atteints, mais il reste, cependant, un exercice de style sans réelle portée pratique, alors que pour d'autres il constitue un outil d'aide à la décision vraiment adapté au réel besoin de quantification des divers éléments de la prise de décision. Bien sûr, ces positions représentent des attitudes extrêmes et de nombreux auteurs avaient des positions plus nuancées mais néanmoins contradictoires.

II nous semble, en fait, que certaines autorités, plutôt que d'adopter une approche du type de celle préconisée par le principe ALARA, ont choisi de fixer des objectifs en termes de limites, à des niveaux suffisamment bas pour qu'il ne soit pas nécessaire de poursuivre les efforts pour réduire encore les expositions lorsque ces objectifs sont atteints. Cette démarche est celle qui prévaut aux États-Unis et en République fédérale d'Allemagne. On peut alors arguer que si l'objectif est suffisamment ambitieux, le principe ALARA n'a plus d'intérêt, car l'on se trouve presque inévitablement au-delà du niveau optimal de protection. Nous pensons que ce dernier argument explique, sur le fond, les conclusions plutôt négatives qui sont apparues dans certaines communications quant à l'efficacité du principe ALARA. Si l'objectif que l'on doit atteindre est déjà au-delà du niveau de protection qu'une analyse de type ALARA démontrerait comme raisonnable, 
alors il n'y a aucun intérêt à développer de telles analyses qui n'apporteront aucune information supplémentaire, à moins de mettre l'accent sur le fait que l'on est allé trop loin, ce dont certains ne veulent justement pas entendre parler. Ce raisonnement était en tout cas latent dans plusieurs discussions.

A l'opposé, l'analyse réalisée au Royaume-Uni concernant la conception du projet de réacteur à eau pressurisée (REP) pour le site de Sizewell est, sans conteste, l'exemple le plus poussé d'intégration du principe ALARA qui fut présenté au cours de ces journées. Cette étude réalisée par le concepteur (Central electricity generating board) [27] s'appuie sur des analyses coût-bénéfice pour apporter aux décideurs - dans ce cas les autorités de sûreté - des éléments quantifiés quant à l'opportunité d'introduire des modifications de conception concernant la protection radiologique des travailleurs et du public, par rapport aux réacteurs REP standards déjà en exploitation. Les autorités de sûreté ont reconnu que ces analyses étaient appropriées et les ont retenues pour déterminer leurs propres conclusions quant au bien fondé des modifications. II s'agit là d'une application assez exemplaire du principe ALARA.

L'intégration de ce principe reste de toute façon un problème délicat, et il convient de distinguer plusieurs catégories d'analyses : celles qui sont effectuées a posteriori pour vérifier la conformité des choix avec le principe, celles plus intéressantes menées a priori pour aider les décideurs dans leur choix. Parmi ces dernières, il est évident que les études réalisées au niveau de la conception des installations présentent l'avantage d'une souplesse qui n'est plus possible au niveau de l'exploitation. Enfin, il faut également le souligner, les études de type ALARA ne sont intéressantes que pour aider des décisions présentant une certaine complexité au niveau de la diversité des options envisageables, et surtout lorsque les enjeux économiques sont significatifs (1). La grande majorité des décisions concernant la radioprotection quotidienne et pratique doit rester, en principe, en dehors du champ des études d'évaluation dès lors que les options de base au niveau de la conception des installations sont conformes avec le principe ALARA.

Des études particulières doivent être entreprises pour mieux cerner le domaine de validité des études d'évaluation afin de préciser quand et comment de telles études peuvent intervenir au niveau des processus décisionnels. En tout cas, il ne faisait aucun doute dans les esprits des participants, que le principe ALARA ne devrait avoir aucun caractère systématique, de même que les résultats auxquels il conduit ne devraient présenter, comme on l'a déjà souligné, aucun caractère obligatoire.

\subsection{La constitution de bases de données adaptées}

II est évident que des données adaptées doivent être disponibles pour alimenter les études de type ALARA. Ce point n'a, hélas, pas reçu tout l'intérêt qu'il mérite jusqu'ici et de nombreuses discussions

(1) Voir dans ce numéro, p.295, l'article de A. Oudiz et J. Lombard sur ces aspects. 
stériles sur la soi-disant complexité du principe ALARA auraient sûrement pu être évitées si ce problème avait été reconnu dès le départ comme l'un des obstacles majeurs à la mise en application du principe.

En effet, l'essentiel des données disponibles jusqu'à une époque très récente provient soit de la dosimétrie réglementaire individuelle pour les travailleurs, soit des mesures de radioactivité ambiante dans l'environnement, dont les objectifs sont la vérification du respect des limites de dose et le contrôle a posteriori des sources. Ces données sont pratiquement inutilisables pour les études d'évaluation de la radioprotection dans la mesure où elles ne fournissent aucune information sur la relation entre les sources et les doses reçues. L'introduction du principe ALARA devrait opérer un changement de perspective dans le recueil des données dosimétriques surtout en ce qui concerne l'exposition professionnelle. Aux relevés traditionnels de doses individuelles devraient s'ajouter, quasi systématiquement, l'établissement de cartographies de débits de dose ambiants et au contact des principaux composants radioactifs des installations ainsi que le relevé des durées et des fréquences d'interventions du personnel. Un mouvement commence à se dessiner dans ce sens en France [1] mais, dans l'ensemble, les études présentées au cours du séminaire s'appuient sur des données qu'il a fallu rechercher au coup par coup au fur et à mesure des besoins de l'analyse. En ce qui concerne l'exposition professionnelle, certaines études ont été réalisées avec des données issues de l'expérience acquise [18] mais la plupart des auteurs ont, cependant, eu recours à des estimations prévisionnelles, parfois assez grossières, pratiquées à partir de synthèses de données éparses [27, 29]. Les observations directes et les mesures restent très rares dans le cas de la radioactivité dispersée dans l'environnement [17] et la plupart des études sont basées sur des modèles, comme nous l'avons déjà souligné. II faut également noter que les analyses de type ALARA requièrent, outre des données dosimétriques, des données d'ordre plus général concernant les activités à l'origine des expositions, comme les trafics routiers et les fréquences d'accidents de poids lourds pour évaluer les risques associés au transport de matières radioactives par route [16] ou encore les taux d'occupation des lits d'hôpitaux pour évaluer l'impact d'une modification de la protection des patients et du personnel dans une unité de radiothérapie [11].

Le recueil de données adaptées est primordial et un effort doit être fait pour développer, dans l'avenir, des bases de données fiables permettant d'évaluer la protection en terme technico-économiques.

\subsection{De l'usage des valeurs monétaires de l'unité de dose}

La plupart des auteurs ont utilisé des valeurs monétaires de l'unité de dose collective, bien que, dans de nombreux cas, il n'était pas clairement spécifié s'il s'agissait de "pures valeurs d'alpha" ou si ces valeurs n'incluaient pas également implicitement une composante "bêta" (voir § 3.3). La distinction affirmée par les auteurs était plûtot relative à celle que l'on peut faire entre valeurs de l'homme-sievert fixées a priori $[2,11,17,26,28]$ et valeurs déterminées a posteriori $[3,10,18,30]$. 
Par valeurs a priori, il faut comprendre celles obtenues à partir de calculs reposant sur un cadre théorique préétabli, comme la théorie économique du prix de la vie humaine, et, ensuite appliquées systématiquement dans tous les cas de figure. A l'opposé, les valeurs de l'unité de dose peuvent être dérivées de l'analyse de décisions qui ont déjà été prises, ou de décisions qui sont jugées comme raisonnables. Puisque pour toute décision concernant la protection correspond un coût et un certain bénéfice, qui s'exprime en termes de dose évitée, il est en effet possible de déterminer directement le coût implicite de l'unité de dose évitée en calculant le rapport coût/dose évitée. Cette dernière démarche, nettement plus pratique, présente évidemment l'inconvénient de ne pas fournir des valeurs qui sont toujours représentatives, dans la mesure où les décisions retenues ont pu être influencées par des facteurs qui ne relèvent ni des coûts, ni de l'exposition, introduisant de la sorte des distorsions.

Le tableau I en annexe récapitule les valeurs monétaires de l'unité de dose relevées dans les différentes communications. On peut noter une certaine cohérence entre les valeurs utilisées, qu'il s'agisse des valeurs a priori ou a posteriori. Pour ces dernières, seules celles relatives à la protection des patients [11] et du public dans le cas d'événements probabilistes [16] sont notablement divergentes. Le tableau II fournit le détail du système de valeurs monétaires fixées a priori et combinant à la fois les dimensions "alpha" et "bêta" proposées par le NRPB pour l'évaluation de la protection des travailleurs et du public.

Des discussions relatives à l'utilisation des valeurs monétaires de l'unité de dose, il est surtout ressorti le besoin, parmi les praticiens, d'une uniformisation des valeurs devant être utilisées dans les différents pays pour les analyses ALARA et, tout particulièrement celles devant être utilisées dans le cas des pollutions transfrontières.

\subsection{Le développement des méthodes d'aide à la décision}

Comme nous l'avons déjà souligné, la plupart des développements méthodologiques concernant le principe ALARA ont été consacrés à l'analyse coût-bénéfice, selon la ligne tracée par la CIPR. Bien que cette démarche présente un intérêt certain, en particulier sa facilité de mise en œuvre, lorsqu'il s'agit de problèmes relativement simples - la plupart des études de cas présentées au cours de ces journées de travail sont là pour le démontrer - elle atteint rapidement ses limites lorsque le cadre décisionnel présente de multiples dimensions dont on ne peut faire abstraction. II est certain que les analyses de type multicritères mieux adaptées dans ce cas sont, cependant, plus complexes à mettre en œuvre, et demandent sûrement un travail méthodologique préalable pour définir des procédures simplifiées pour les praticiens de la radioprotection. En fait, il serait souhaitable que, dans les années à venir, un effort particulier soit consacré par les équipes qui jusqu'ici se sont attachées à développer la méthodologie du principe ALARA, à l'investigation des interactions qui existent forcément entre les diverses méthodes d'aide à la décision, pour aboutir à terme à une intégration de l'analyse coût-bénéfice dans un système plus large à caractère multicritère. 
Le travail déjà réalisé sur l'analyse coût-bénéfice serait ainsi préservé, tout en dépassant les limites propres à cette méthode. Ceci dit, il n'est pas impossible d'envisager que le résultat de ces recherches n'aboutisse à mettre à la disposition des praticiens une palette d'outils simples et mieux adaptés à la complexité des problèmes auxquels ils sont parfois confrontés.

\subsection{La pondération des critères}

L'une des difficultés majeures au niveau de la mise en œuvre de toute méthode d'aide à la décision, y compris bien sûr l'analyse coût-bénéfice, réside dans le problème de la pondération des critères. Si l'on veut introduire assez de souplesse dans les méthodes décisionnelles pour justement permettre à l'analyste l'usage de différents jeux de pondération entre les critères, alors il faut élargir le système. C'est dans cette perspective que la CIPR a introduit l'idée du terme "bêta" dans la formule initiale de la méthode coût-bénéfice qu'elle préconisait (voir $\S 3.3$ ). Cette modification du système est, cependant, trop récente pour que des solutions pratiques aient eu le temps d'être développées.

Les pondérations qui semblent devoir être introduites dans les études d'évaluation de la protection sont celles relatives à la distribution des doses individuelles, celles concernant le fait qu'il s'agit de l'exposition du public ou des travailleurs, celles, enfin, concernant la distribution des doses dans le temps et les doses associées à des événements probabilistes. II faudra donc fractionner la dose collective totale en autant de sous-parties correspondant à ces diverses dimensions et définir pour chacune d'elle un ensemble de classes pour caractériser au mieux les distributions de dose considérées. La question qui reste tout à fait ouverte pour le moment est celle du poids à accorder à ces diverses sous-parties de la dose collective totale et, à l'intérieur de chaque partie, aux diverses classes. II n'existe pas encore de véritable consensus parmi ceux qui réfléchissent à ce problème ni sur la technique à adopter pour définir le système des classes relatives à chaque dimension, ni sur la valeur des coefficients de pondération qui pourraient y être attachés. On peut, cependant, noter que plusieurs des exemples présentés abordaient ce problème explicitement, en particulier ceux pour lesquels les actions de protection analysées ont une influence à la fois sur l'exposition du public et sur celle des travailleurs [9, 11, 27].

Le problème de la pondération des doses individuelles a d'ores et déjà été introduit dans le système coût-bénéfice recommandé par le NRPB et qui est encore actuellement en discussion. L'un des résultats le plus significatif de la réflexion menée par le NRPB sur les différences de poids à accorder aux doses individuelles en fonction de leur niveau, est, sans conteste, l'introduction du concept de doses de minimis, c'est-à-dire de doses considérées suffisamment faibles pour être écartées de l'analyse. La définition précise du concept de dose de minimis fait encore l'objet de discussions, mais néanmoins le concept apparaît dans certains travaux.

La pondération des doses délivrées dans le temps (déjà évoquée dans le $\S 3.4$ ) et qui peut être abordée soit en utilisant les techniques de l'actualisation du coût du détriment ou bien de la troncature de la dose collective au-delà d'un certain point, est un autre problème délicat. Dans 
la procédure ALARA, qui consiste à choisir entre diverses options de protection, il apparaît logique de cesser de considérer la distribution des doses comme un paramètre significatif, dès lors que cette dernière n'est plus influencée par la différence entre les options. C'est ce que l'on peut généralement constater dans le cas des doses délivrées par les radionucléides de longue période qui ont atteint un certain niveau de dispersion globale dans l'environnement et qui ne sont plus modifiables par les options que l'on peut envisager. L'argument de l'incertitude sur les évaluations des expositions qui tend à s'accroître très vite en fonction du temps, de même que la préférence pour le présent qui caractérise le comportement individuel et social dans nos sociétés fournissent, par ailleurs, une justification à la pondération du coût du détriment futur.

Le besoin de développer une méthodologie et des techniques pour répondre aux divers compromis auxquels les décideurs sont confrontés dans leur choix de radioprotection a été reconnu par tous les auteurs et il convient donc d'encourager les initiatives allant dans ce sens, d'autant plus qu'aucune convergence ne semble se dessiner dans les travaux ayant déjà abordé le sujet.

\subsection{Les contraintes additionnelles}

La procédure d'évaluation des actions de protection selon le principe ALARA s'effectue sous contraintes, les plus évidentes étant, bien entendu, les limites de doses individuelles. D'autres contraintes existent dans certains cas, de nature technique ou économique comme une contrainte de budget. On peut également rencontrer des situations où des contraintes additionnelles relatives aux niveaux d'exposition sont imposées par les autorités ou plus simplement par ceux qui développent les analyses. Lorsque de telles contraintes s'ajoutent aux contraintes normales sur les limites de dose individuelles, il faut alors interpréter les résultats des évaluations avec une certaine réserve dans la mesure où ces contraintes supplémentaires ont pu éliminer des options qui, en leur absence, auraient été "optimales". Plusieurs études de cas présentées incluaient ce type de contraintes additionnelles soit sur les doses individuelles, soit sur les doses collectives $[3,10,27]$. II conviendrait d'analyser de façon plus approfondie l'impact de telles contraintes sur la procédure ALARA et ses résultats plutôt que d'en déduire abusivement que cette procédure est alors sans intérêt.

\section{CONCLUSION}

A l'époque où les conditions de sécurité des travailleurs étaient encore médiocres et les préoccupations du public pour les problèmes d'environnement quasi inexistantes, il était légitime pour ceux chargés d'améliorer le niveau général de protection des populations de se fixer des objectifs assez ambitieux sans se soucier outre mesure des coûts ni même des difficultés pratiques, sachant qu'inévitablement les responsables des activités à l'origine des nuisances se chargeraient bien de résister à ce qui pouvait apparaître comme des excès de zèle. Au fil des années, cependant, la pression des travailleurs mais aussi du public pour l'amélioration de leur environnement 
n'a cessé de s'accroître, renforçant par là même le pouvoir des autorités régulatrices. Accompagnant ce mouvement, les préoccupations économiques ont également vu leur importance s'étendre dans la mesure où la protection et la sûreté des populations absorbaient une part de moins en moins négligeable des ressources sociales.

Dans un tel contexte, si l'on veut éviter que le mouvement irréversible vers une protection toujours accrue des populations, prise en charge par des autorités et des administrations toujours plus puissantes, n'aboutisse à un accaparement exagéré des ressources disponibles, il faut alors soit donner à ceux qui mettent en œuvre la protection sur le terrain des moyens de résister - ce qui n'est pas du tout dans l'optique des réglementations actuelles - soit faire en sorte que le système de protection génère de lui-même une limite. C'est là, nous semble-t-il, l'idée fondamentale qui justifie le principe ALARA et en fait sa force et qui devrait retenir l'attention de ceux qui veulent promouvoir ce principe. On peut noter que cette idée de mettre ainsi en balance le niveau de risque résiduel et le coût de protection n'est pas nouvelle et qu'on la retrouve exprimée sous des formes plus ou moins voisines dans de nombreuses réglementations.

Dans l'ensemble, les participants aux journées de travail se sont trouvés d'accord sur le fait que trop souvent le principe ALARA est encore perçu comme un outil pour abaisser toujours plus les niveaux de risques résiduels, en confondant complètement, de surcroît, le principe et l'un des outils possible de sa mise en œuvre, en l'occurence l'analyse coût-bénéfice. L'optimisation de la protection n'est pas la minimisation des expositions et l'analyse coût-bénéfice est un outil qui n'est pas toujours, comme on l'a vu, le mieux adapté pour répondre aux besoins de radioprotectionnistes soucieux d'évaluer les actions de protection qu'ils envisagent. Cette mauvaise compréhension n'est certainement pas le fruit du hasard, mais le résultat de trop nombreuses présentations répétées au fil des années qui n'ont pas su mettre assez l'accent sur l'idée essentielle qui anime le principe ALARA et se sont cantonnées dans la reprise du modèle coût-bénéfice de la CIPR.

Ce point étant rappelé, la principale conclusion que l'on peut tirer de ces journées, c'est que, désormais, dans de nombreux pays, des équipes appliquent des techniques quantitatives pour mettre en œuvre le principe ALARA ; les communications présentées en sont la démonstration la plus évidente. Si des réticences subsistent encore chez certains quant à l'utilité du principe, c'est, nous semble-t-il, parce que les contraintes imposées à la protection sont telles qu'elles ont éliminé la nécessité de vérifier que les niveaux de protection satisfont au principe. En fait, pour la majorité des participants, il ne fait plus de doute que la quantification des actions de protection dans un cadre formel conforme à l'idée mise en avant par la CIPR est utile : elle permet de clarifier les diverses dimensions du problème étudié et fournit ainsi aux décideurs tous les éléments pour mieux rechercher le meilleur compromis possible entre ces éléments contradictoires. Pour l'avenir, l'effort doit porter sur le développement de techniques d'aide à la décision mieux adaptées aux situations complexes et la définition de valeurs traduisant l'importance devant être accordée aux diverses dimensions de la radioprotection. L'usage généralisé de ces techniques et ces valeurs explicitement choisies permettront d'introduire 
dans les décisions une certaine cohérence qui ne peut, à terme, que déboucher sur un traitement équitable de la protection dans les diverses activités où elle est présente.

\section{ANNEXE}

TABLEAU I

Valeurs monétaires de l'unité de dose collective utilisées dans les études de cas

\begin{tabular}{|l|c|c|l|}
\hline \multirow{2}{*}{ Auteurs } & \multicolumn{2}{|c|}{$\begin{array}{c}\text { Coût de l'unité de dose collective } \\
\text { (US \$-1980-homme-sievert) }\end{array}$} & \\
& A priori & A posteriori & Population \\
\hline $\begin{array}{l}\text { Erixon [10] } \\
\text { Jeanson [18] }\end{array}$ & & $\begin{array}{c}15000-100000 \\
20000-500000\end{array}$ & Travailleurs \\
$\begin{array}{l}\text { Hock [13] } \\
\text { Hock [14] }\end{array}$ & 7500 & $13000-750000$ & " \\
$\begin{array}{l}\text { Wahlströem [30] } \\
\text { Oudiz [26] }\end{array}$ & & 100000 & $\begin{array}{l}\text { Public } \\
\text { Travailleurs } \\
\text { Dobici [9] } \\
\text { Bonka [4] } \\
\text { Benassai [3] } \\
\text { Hunt [17] } \\
\text { Hubert [16] }\end{array}$ \\
\hline
\end{tabular}

* Mesure de sûreté.

TABLEAU ॥

Système de valeurs monétaires recommandé par le NRPB

\begin{tabular}{|c|c|c|c|}
\hline \multicolumn{2}{|c|}{ Public } & \multicolumn{2}{c|}{ Travailleurs } \\
\hline $\begin{array}{c}\text { Niveau } \\
\text { de dose } \\
\text { individuelle } \\
\text { (mSv) }\end{array}$ & $\begin{array}{c}\text { Coût de } \\
\text { I'homme-sievert } \\
\text { (US \$-1980) }\end{array}$ & $\begin{array}{c}\text { Niveau } \\
\text { de dose } \\
\text { individuelle } \\
\text { (mSv) }\end{array}$ & $\begin{array}{c}\text { Coût de } \\
\text { I'homme-sievert } \\
\text { (US \$-1980) }\end{array}$ \\
\hline$<0.05$ & 3000 & $<5$ & 6000 \\
$0.05-0.5$ & 15000 & $5-15$ & 30000 \\
$0.5-5$ & 75000 & $15-50$ & 150000 \\
\hline
\end{tabular}




\section{RÉFÉRENCES}

[1] AYE L. Evolution des doses reçues par les travailleurs dans les centrales nucléaires PWR d'EdF. Radioprotection, 1983, 18 (2) 65-78.

[2] BARNES D.S., BROWN M.L., MEGGITT G.C. Optimization of an LMFBR reprocessing plant for routine emissions. In : Radiation protection optimization "As Low As Reasonably Achievable", 2 nd European scientific seminar, Luxembourg, 8-9 Nov. 1983 (JOLIVET A., SINNAEVE J., Eds). EUR-9173. Luxembourg : Communautés européennes, 1984, 331-352.

[3] BENASSAI S., BRAMATI L. Optimization of radiation protection in nuclear power plants in Italy. In: Radiation protection optimization "As Low As Reasonably Achievable", 2 nd European scientific seminar, Luxembourg, 8-9 Nov. 1983 (JOLIVET A., SINNAEVE J., Eds). EUR-9173. Luxembourg: Communautés européennes, 1984, 251-257.

[4] BONKA H., HORN H.G. Optimization of the retention of radioactive material from the airbone effluents of reprocessing plants. In : Radiation protection optimization "As Low As Reasonably Achievable", 2 nd European scientific seminar, Luxembourg, 8-9 Nov. 1983 (JOLIVET A., SINNAEVE J., Eds). EUR-9173. Luxembourg: Communautés européennes, 1984, 353-377.

[5] Commission internationale de protection radiologique (CIPR). Recommandations Publication CIPR n०26. Oxford: Pergamon press, 1980.

[6] Commission internationale de protection radiologique (CIPR). Les implications des recommandations de la Commission de maintenir les doses aux valeurs les plus faibles qu'il soit possible d'atteindre sans difficulté. Publication CIPR 22. Fontenayaux-Roses : CEA, 1974 et Implications of Commission recommandations that doses be kept as low as readily achievable. ICRP Publication 22. Oxford : Pergamon press, 1973.

[7] International Commission on Radiological Protection. Cost-benefit analysis in the optimization of radiation protection. ICRP Publication 37. Oxford: Pergamon press, 1983.

[8] Communautés européennes (Conseil). Directive du 15 juillet 1980, portant modification des directives fixant les normes de base relatives à la protection sanitaire de la population et des travailleurs contre les dangers résultant des rayonnements ionisants. J. Off. Comm. Europ., 1980, L. 246.

[9] DOBICI F., FRITTELLI L., BELLI M., BIANCOTTO R., TACCONI A., NEGRI F. Experience in optimizing radiation protection in medical application of radiation. In : Radiation protection optimization "As Low As Reasonably Achievable", 2 nd European scientific seminar, Luxembourg, 8-9 Nov. 1983 (JOLIVET A., SINNAEVE J., Eds). EUR-9173. Luxembourg : Communautés européennes, 1984, 299-310.

[10] ERIXON O. Experience gained of dose reducing measures at Ringhals unit 2 during 1975 to 1983 . In : Radiation protection optimization "As Low As Reasonably Achievable", 2 nd European scientific seminar, Luxembourg, 8-9 Nov. 1983 (JOLIVET A., SINNAEVE J., Eds). EUR-9173. Luxembourg: Communautés européennes, 1984, 139-151.

[11] FLEISHMAN A.B., NOTLEY H.M., WILKINSON J.M. Optimization of protection for gynecological radiotherapy. In": Radiation protection optimization "As Low As Reasonably Achievable", 2 nd European scientific seminar, Luxembourg, 8-9 Nov. 1983 (JOLIVET A., SINNAEVE J., Eds). EUR-9173. Luxembourg : Communautés européennes, 1984, 311-327.

[12] HEMMING C.R., KELLY G.N., CHARLES D. The influence of non-linear dose response relationship for late effects on the predict consequences of accidental releases of radioactive material. Rapport NRPB-M-91, 1983. 
alors il n'y a aucun intérêt à développer de telles analyses qui n'apporteront aucune information supplémentaire, à moins de mettre l'accent sur le fait que l'on est allé trop loin, ce dont certains ne veulent justement pas entendre parler. Ce raisonnement était en tout cas latent dans plusieurs discussions.

A l'opposé, l'analyse réalisée au Royaume-Uni concernant la conception du projet de réacteur à eau pressurisée (REP) pour le site de Sizewell est, sans conteste, l'exemple le plus poussé d'intégration du principe ALARA qui fut présenté au cours de ces journées. Cette étude réalisée par le concepteur (Central electricity generating board) [27] s'appuie sur des analyses coût-bénéfice pour apporter aux décideurs - dans ce cas les autorités de sûreté - des éléments quantifiés quant à l'opportunité d'introduire des modifications de conception concernant la protection radiologique des travailleurs et du public, par rapport aux réacteurs REP standards déjà en exploitation. Les autorités de sûreté ont reconnu que ces analyses étaient appropriées et les ont retenues pour déterminer leurs propres conclusions quant au bien fondé des modifications. II s'agit là d'une application assez exemplaire du principe ALARA.

L'intégration de ce principe reste de toute façon un problème délicat, et il convient de distinguer plusieurs catégories d'analyses : celles qui sont effectuées a posteriori pour vérifier la conformité des choix avec le principe, celles plus intéressantes menées a priori pour aider les décideurs dans leur choix. Parmi ces dernières, il est évident que les études réalisées au niveau de la conception des installations présentent l'avantage d'une souplesse qui n'est plus possible au niveau de l'exploitation. Enfin, il faut également le souligner, les études de type ALARA ne sont intéressantes que pour aider des décisions présentant une certaine complexité au niveau de la diversité des options envisageables, et surtout lorsque les enjeux économiques sont significatifs (1). La grande majorité des décisions concernant la radioprotection quotidienne et pratique doit rester, en principe, en dehors du champ des études d'évaluation dès lors que les options de base au niveau de la conception des installations sont conformes avec le principe ALARA.

Des études particulières doivent être entreprises pour mieux cerner le domaine de validité des études d'évaluation afin de préciser quand et comment de telles études peuvent intervenir au niveau des processus décisionnels. En tout cas, il ne faisait aucun doute dans les esprits des participants, que le principe ALARA ne devrait avoir aucun caractère systématique, de même que les résultats auxquels il conduit ne devraient présenter, comme on l'a déjà souligné, aucun caractère obligatoire.

\subsection{La constitution de bases de données adaptées}

II est évident que des données adaptées doivent être disponibles pour alimenter les études de type ALARA. Ce point n'a, hélas, pas reçu tout l'intérêt qu'il mérite jusqu'ici et de nombreuses discussions

(1) Voir dans ce numéro, p.295, l'article de A. Oudiz et J. Lombard sur ces aspects. 
stériles sur la soi-disant complexité du principe ALARA auraient sûrement pu être évitées si ce problème avait été reconnu dès le départ comme l'un des obstacles majeurs à la mise en application du principe.

En effet, l'essentiel des données disponibles jusqu'à une époque très récente provient soit de la dosimétrie réglementaire individuelle pour les travailleurs, soit des mesures de radioactivité ambiante dans l'environnement, dont les objectifs sont la vérification du respect des limites de dose et le contrôle a posteriori des sources. Ces données sont pratiquement inutilisables pour les études d'évaluation de la radioprotection dans la mesure où elles ne fournissent aucune information sur la relation entre les sources et les doses reçues. L'introduction du principe ALARA devrait opérer un changement de perspective dans le recueil des données dosimétriques surtout en ce qui concerne l'exposition professionnelle. Aux relevés traditionnels de doses individuelles devraient s'ajouter, quasi systématiquement, l'établissement de cartographies de débits de dose ambiants et au contact des principaux composants radioactifs des installations ainsi que le relevé des durées et des fréquences d'interventions du personnel. Un mouvement commence à se dessiner dans ce sens en France [1] mais, dans l'ensemble, les études présentées au cours du séminaire s'appuient sur des données qu'il a fallu rechercher au coup par coup au fur et à mesure des besoins de l'analyse. En ce qui concerne l'exposition professionnelle, certaines études ont été réalisées avec des données issues de l'expérience acquise [18] mais la plupart des auteurs ont, cependant, eu recours à des estimations prévisionnelles, parfois assez grossières, pratiquées à partir de synthèses de données éparses [27, 29]. Les observations directes et les mesures restent très rares dans le cas de la radioactivité dispersée dans l'environnement [17] et la plupart des études sont basées sur des modèles, comme nous l'avons déjà souligné. II faut également noter que les analyses de type ALARA requièrent, outre des données dosimétriques, des données d’ordre plus général concernant les activités à l'origine des expositions, comme les trafics routiers et les fréquences d'accidents de poids lourds pour évaluer les risques associés au transport de matières radioactives par route [16] ou encore les taux d'occupation des lits d'hôpitaux pour évaluer l'impact d'une modification de la protection des patients et du personnel dans une unité de radiothérapie [11].

Le recueil de données adaptées est primordial et un effort doit être fait pour développer, dans l'avenir, des bases de données fiables permettant d'évaluer la protection en terme technico-économiques.

\subsection{De l'usage des valeurs monétaires de l'unité de dose}

La plupart des auteurs ont utilisé des valeurs monétaires de l'unité de dose collective, bien que, dans de nombreux cas, il n'était pas clairement spécifié s'ii s'agissait de "pures valeurs d'alpha" ou si ces valeurs n'incluaient pas également implicitement une composante "bêta" (voir § 3.3). La distinction affirmée par les auteurs était plûtot relative à celle que l'on peut faire entre valeurs de l'homme-sievert fixées a priori $[2,11,17,26,28]$ et valeurs déterminées a posteriori $[3,10,18,30]$. 
Par valeurs a priori, il faut comprendre celles obtenues à partir de calculs reposant sur un cadre théorique préétabli, comme la théorie économique du prix de la vie humaine, et, ensuite appliquées systématiquement dans tous les cas de figure. A l'opposé, les valeurs de l'unité de dose peuvent être dérivées de l'analyse de décisions qui ont déjà été prises, ou de décisions qui sont jugées comme raisonnables. Puisque pour toute décision concernant la protection correspond un coût et un certain bénéfice, qui s'exprime en termes de dose évitée, il est en effet possible de déterminer directement le coût implicite de l'unité de dose évitée en calculant le rapport coût/dose évitée. Cette dernière démarche, nettement plus pratique, présente évidemment l'inconvénient de ne pas fournir des valeurs qui sont toujours représentatives, dans la mesure où les décisions retenues ont pu être influencées par des facteurs qui ne relèvent ni des coûts, ni de l'exposition, introduisant de la sorte des distorsions.

Le tableau I en annexe récapitule les valeurs monétaires de l'unité de dose relevées dans les différentes communications. On peut noter une certaine cohérence entre les valeurs utilisées, qu'il s'agisse des valeurs a priori ou a posteriori. Pour ces dernières, seules celles relatives à la protection des patients [11] et du public dans le cas d'événements probabilistes [16] sont notablement divergentes. Le tableau II fournit le détail du système de valeurs monétaires fixées a priori et combinant à la fois les dimensions "alpha" et "bêta" proposées par le NRPB pour l'évaluation de la protection des travailleurs et du public.

Des discussions relatives à l'utilisation des valeurs monétaires de l'unité de dose, il est surtout ressorti le besoin, parmi les praticiens, d'une uniformisation des valeurs devant être utilisées dans les différents pays pour les analyses ALARA et, tout particulièrement celles devant être utilisées dans le cas des pollutions transfrontières.

\subsection{Le développement des méthodes d'aide à la décision}

Comme nous l'avons déjà souligné, la plupart des développements méthodologiques concernant le principe ALARA ont été consacrés à l'analyse coût-bénéfice, selon la ligne tracée par la CIPR. Bien que cette démarche présente un intérêt certain, en particulier sa facilité de mise en œuvre, lorsqu'il s'agit de problèmes relativement simples - la plupart des études de cas présentées au cours de ces journées de travail sont là pour le démontrer - elle atteint rapidement ses limites lorsque le cadre décisionnel présente de multiples dimensions dont on ne peut faire abstraction. II est certain que les analyses de type multicritères mieux adaptées dans ce cas sont, cependant, plus complexes à mettre en œuvre, et demandent sûrement un travail méthodologique préalable pour définir des procédures simplifiées pour les praticiens de la radioprotection. En fait, il serait souhaitable que, dans les années à venir, un effort particulier soit consacré par les équipes qui jusqu'ici se sont attachées à développer la méthodologie du principe ALARA, à l'investigation des interactions qui existent forcément entre les diverses méthodes d'aide à la décision, pour aboutir à terme à une intégration de l'analyse coût-bénéfice dans un système plus large à caractère multicritère. 
Le travail déjà réalisé sur l'analyse coût-bénéfice serait ainsi préservé, tout en dépassant les limites propres à cette méthode. Ceci dit, il n'est pas impossible d'envisager que le résultat de ces recherches n'aboutisse à mettre à la disposition des praticiens une palette d'outils simples et mieux adaptés à la complexité des problèmes auxquels ils sont parfois confrontés.

\subsection{La pondération des critères}

L'une des difficultés majeures au niveau de la mise en œuvre de toute méthode d'aide à la décision, y compris bien sûr l'analyse coût-bénéfice, réside dans le problème de la pondération des critères. Si l'on veut introduire assez de souplesse dans les méthodes décisionnelles pour justement permettre à l'analyste l'usage de différents jeux de pondération entre les critères, alors il faut élargir le système. C'est dans cette perspective que la CIPR a introduit l'idée du terme "bêta" dans la formule initiale de la méthode coût-bénéfice qu'elle préconisait (voir $\S 3.3$ ). Cette modification du système est, cependant, trop récente pour que des solutions pratiques aient eu le temps d'être développées.

Les pondérations qui semblent devoir être introduites dans les études d'évaluation de la protection sont celles relatives à la distribution des doses individuelles, celles concernant le fait qu'il s'agit de l'exposition du public ou des travailleurs, celles, enfin, concernant la distribution des doses dans le temps et les doses associées à des événements probabilistes. II faudra donc fractionner la dose collective totale en autant de sous-parties correspondant à ces diverses dimensions et définir pour chacune d'elle un ensemble de classes pour caractériser au mieux les distributions de dose considérées. La question qui reste tout à fait ouverte pour le moment est celle du poids à accorder à ces diverses sous-parties de la dose collective totale et, à l'intérieur de chaque partie, aux diverses classes. II n'existe pas encore de véritable consensus parmi ceux qui réfléchissent à ce problème ni sur la technique à adopter pour définir le système des classes relatives à chaque dimension, ni sur la valeur des coefficients de pondération qui pourraient y être attachés. On peut, cependant, noter que plusieurs des exemples présentés abordaient ce problème explicitement, en particulier ceux pour lesquels les actions de protection analysées ont une influence à la fois sur l'exposition du public et sur celle des travailleurs [9, 11, 27].

Le problème de la pondération des doses individuelles a d'ores et déjà été introduit dans le système coût-bénéfice recommandé par le NRPB et qui est encore actuellement en discussion. L'un des résultats le plus significatif de la réflexion menée par le NRPB sur les différences de poids à accorder aux doses individuelles en fonction de leur niveau, est, sans conteste, l'introduction du concept de doses de minimis, c'est-à-dire de doses considérées suffisamment faibles pour être écartées de l'analyse. La définition précise du concept de dose de minimis fait encore l'objet de discussions, mais néanmoins le concept apparaît dans certains travaux.

La pondération des doses délivrées dans le temps (déjà évoquée dans le $\S 3.4$ ) et qui peut être abordée soit en utilisant les techniques de l'actualisation du coût du détriment ou bien de la troncature de la dose collective au-delà d'un certain point, est un autre problème délicat. Dans 
la procédure ALARA, qui consiste à choisir entre diverses options de protection, il apparaît logique de cesser de considérer la distribution des doses comme un paramètre significatif, dès lors que cette dernière n'est plus influencée par la différence entre les options. C'est ce que l'on peut généralement constater dans le cas des doses délivrées par les radionucléides de longue période qui ont atteint un certain niveau de dispersion globale dans l'environnement et qui ne sont plus modifiables par les options que l'on peut envisager. L'argument de l'incertitude sur les évaluations des expositions qui tend à s'accroître très vite en fonction du temps, de même que la préférence pour le présent qui caractérise le comportement individuel et social dans nos sociétés fournissent, par ailleurs, une justification à la pondération du coût du détriment futur.

Le besoin de développer une méthodologie et des techniques pour répondre aux divers compromis auxquels les décideurs sont confrontés dans leur choix de radioprotection a été reconnu par tous les auteurs et il convient donc d'encourager les initiatives allant dans ce sens, d'autant plus qu'aucune convergence ne semble se dessiner dans les travaux ayant déjà abordé le sujet.

\subsection{Les contraintes additionnelles}

La procédure d'évaluation des actions de protection selon le principe ALARA s'effectue sous contraintes, les plus évidentes étant, bien entendu, les limites de doses individuelles. D'autres contraintes existent dans certains cas, de nature technique ou économique comme une contrainte de budget. On peut également rencontrer des situations où des contraintes additionnelles relatives aux niveaux d'exposition sont imposées par les autorités ou plus simplement par ceux qui développent les analyses. Lor'sque de telles contraintes s'ajoutent aux contraintes normales sur les limites de dose individuelles, il faut alors interpréter les résultats des évaluations avec une certaine réserve dans la mesure où ces contraintes supplémentaires ont pu éliminer des options qui, en leur absence, auraient été "optimales". Plusieurs études de cas présentées incluaient ce type de contraintes additionnelles soit sur les doses individuelles, soit sur les doses collectives $[3,10,27]$. II conviendrait d'analyser de façon plus approfondie l'impact de telles contraintes sur la procédure ALARA et ses résultats plutôt que d'en déduire abusivement que cette procédure est alors sans intérêt.

\section{CONCLUSION}

A l'époque où les conditions de sécurité des travailleurs étaient encore médiocres et les préoccupations du public pour les problèmes d'environnement quasi inexistantes, il était légitime pour ceux chargés d'améliorer le niveau général de protection des populations de se fixer des objectifs assez ambitieux sans se soucier outre mesure des coûts ni même des difficultés pratiques, sachant qu'inévitablement les responsables des activités à l'origine des nuisances se chargeraient bien de résister à ce qui pouvait apparaître comme des excès de zèle. Au fil des années, cependant, la pression des travailleurs mais aussi du public pour l'amélioration de leur environnement 
n'a cessé de s'accroître, renforçant par là même le pouvoir des autorités régulatrices. Accompagnant ce mouvement, les préoccupations économiques ont également vu leur importance s'étendre dans la mesure où la protection et la sûreté des populations absorbaient une part de moins en moins négligeable des ressources sociales.

Dans un tel contexte, si l'on veut éviter que le mouvement irréversible vers une protection toujours accrue des populations, prise en charge par des autorités et des administrations toujours plus puissantes, n'aboutisse à un accaparement exagéré des ressources disponibles, il faut alors soit donner à ceux qui mettent en œuvre la protection sur le terrain des moyens de résister - ce qui n'est pas du tout dans l'optique des réglementations actuelles - soit faire en sorte que le système de protection génère de lui-même une limite. C'est là, nous semble-t-il, l'idée fondamentale qui justifie le principe ALARA et en fait sa force et qui devrait retenir l'attention de ceux qui veulent promouvoir ce principe. On peut noter que cette idée de mettre ainsi en balance le niveau de risque résiduel et le coût de protection n'est pas nouvelle et qu'on la retrouve exprimée sous des formes plus ou moins voisines dans de nombreuses réglementations.

Dans l'ensemble, les participants aux journées de travail se sont trouvés d'accord sur le fait que trop souvent le principe ALARA est encore perçu comme un outil pour abaisser toujours plus les niveaux de risques résiduels, en confondant complètement, de surcroît, le principe et l'un des outils possible de sa mise en œuvre, en l'occurence l'analyse coût-bénéfice. L'optimisation de la protection n'est pas la minimisation des expositions et l'analyse coût-bénéfice est un outil qui n'est pas toujours, comme on l'a vu, le mieux adapté pour répondre aux besoins de radioprotectionnistes soucieux d'évaluer les actions de protection qu'ils envisagent. Cette mauvaise compréhension n'est certainement pas le fruit du hasard, mais le résultat de trop nombreuses présentations répétées au fil des années qui n'ont pas su mettre assez l'accent sur l'idée essentielle qui anime le principe ALARA et se sont cantonnées dans la reprise du modèle coût-bénéfice de la CIPR.

Ce point étant rappelé, la principale conclusion que l'on peut tirer de ces journées, c'est que, désormais, dans de nombreux pays, des équipes appliquent des techniques quantitatives pour mettre en œuvre le principe ALARA ; les communications présentées en sont la démonstration la plus évidente. Si des réticences subsistent encore chez certains quant à l'utilité du principe, c'est, nous semble-t-il, parce que les contraintes imposées à la protection sont telles qu'elles ont éliminé la nécessité de vérifier que les niveaux de protection satisfont au principe. En fait, pour la majorité des participants, il ne fait plus de doute que la quantification des actions de protection dans un cadre formel conforme à l'idée mise en avant par la CIPR est utile : elle permet de clarifier les diverses dimensions du problème étudié et fournit ainsi aux décideurs tous les éléments pour mieux rechercher le meilleur compromis possible entre ces éléments contradictoires. Pour l'avenir, l'effort doit porter sur le développement de techniques d'aide à la décision mieux adaptées aux situations complexes et la définition de valeurs traduisant l'importance devant être accordée aux diverses dimensions de la radioprotection. L'usage généralisé de ces techniques et ces valeurs explicitement choisies permettront d'introduire 
dans les décisions une certaine cohérence qui ne peut, à terme, que déboucher sur un traitement équitable de la protection dans les diverses activités où elle est présente.

\section{ANNEXE}

TABLEAU I

Valeurs monétaires de l'unité de dose collective utilisées dans les études de cas

\begin{tabular}{|c|c|c|c|}
\hline \multirow{2}{*}{ Auteurs } & \multicolumn{2}{|c|}{$\begin{array}{l}\text { Coût de l'unité de dose collective } \\
\text { (US } \$-1980-\text { homme-sievert) }\end{array}$} & \multirow{2}{*}{ Population } \\
\hline & A priori & A posteriori & \\
\hline $\begin{array}{l}\text { Erixon [10] } \\
\text { Jeanson [18] } \\
\text { Hock [13] } \\
\text { Hock [14] } \\
\text { Wahlströem [30] } \\
\text { Oudiz [26] } \\
\text { Dobici [9] } \\
\text { Bonka [4] } \\
\text { Benassai [3] } \\
\text { Hunt [17] } \\
\text { Hubert [16] }\end{array}$ & $\begin{array}{r}7500 \\
3000-75000\end{array}$ & $\begin{array}{c}15000-100000 \\
20000-500000 \\
13000-750000 \\
100000 \\
60000 \\
2000-13000 \\
20000-93000 \\
5000000^{\star}\end{array}$ & $\begin{array}{l}\text { Travailleurs } \\
\qquad " \\
\text { Public" } \\
\text { Travailleurs } \\
\text { " } \\
\text { Patients } \\
\text { Public } \\
\text { Travailleurs } \\
\text { Public " }\end{array}$ \\
\hline
\end{tabular}

* Mesure de sûreté.

TABLEAU ॥

Système de valeurs monétaires recommandé par le NRPB

\begin{tabular}{|c|c|c|c|}
\hline \multicolumn{2}{|c|}{ Public } & \multicolumn{2}{c|}{ Travailleurs } \\
\hline $\begin{array}{c}\text { Niveau } \\
\text { de dose } \\
\text { individuelle } \\
\text { (mSv) }\end{array}$ & $\begin{array}{c}\text { Coût de } \\
\text { l'homme-sievert } \\
\text { (US \$-1980) }\end{array}$ & $\begin{array}{c}\text { Niveau } \\
\text { de dose } \\
\text { individuelle } \\
\text { (mSv) }\end{array}$ & $\begin{array}{c}\text { Coût de } \\
\text { l'homme-sievert } \\
\text { (US \$-1980) }\end{array}$ \\
\hline$<0.05$ & 3000 & $<5$ & 6000 \\
$0.05-0.5$ & 15000 & $5-15$ & 30000 \\
$0.5-5$ & 75000 & $15-50$ & 150000 \\
\hline
\end{tabular}




\section{RÉFÉRENCES}

[1] AYE L. Evolution des doses reçues par les travailleurs dans les centrales nucléaires PWR d'EdF. Radioprotection, 1983, 18 (2) 65-78.

[2] BARNES D.S., BROWN M.L., MEGGITT G.C. Optimization of an LMFBR reprocessing plant for routine emissions. In : Radiation protection optimization "As Low As Reasonably Achievable", 2 nd European scientific seminar, Luxembourg, 8-9 Nov. 1983 (JOLIVET A., SINNAEVE J., Eds). EUR-9173. Luxembourg : Communautés européennes, 1984, 331-352.

[3] BENASSAI S., BRAMATI L. Optimization of radiation protection in nuclear power plants in Italy. In: Radiation protection optimization "As Low As Reasonably Achievable", 2 nd European scientific seminar, Luxembourg, 8-9 Nov. 1983 (JOLIVET A., SINNAEVE J., Eds). EUR-9173. Luxembourg: Communautés européennes, 1984, 251-257.

[4] BONKA H., HORN H.G. Optimization of the retention of radioactive material from the airbone effluents of reprocessing plants. In : Radiation protection optimization "As Low As Reasonably Achievable", 2 nd European scientific seminar, Luxembourg, 8-9 Nov. 1983 (JOLIVET A., SINNAEVE J., Eds). EUR-9173. Luxembourg: Communautés européennes, 1984, 353-377.

[5] Commission internationale de protection radiologique (CIPR). Recommandations Publication CIPR n॰26. Oxford: Pergamon press, 1980.

[6] Commission internationale de protection radiologique (CIPR). Les implications des recommandations de la Commission de maintenir les doses aux valeurs les plus faibles qu'il soit possible d'atteindre sans difficulté. Publication CIPR 22. Fontenayaux-Roses : CEA, 1974 et Implications of Commission recommandations that doses be kept as low as readily achievable. ICRP Publication 22. Oxford : Pergamon press, 1973.

[7] International Commission on Radiological Protection. Cost-benefit analysis in the optimization of radiation protection. ICRP Publication 37. Oxford: Pergamon press, 1983.

[8] Communautés européennes (Conseil). Directive du 15 juillet 1980, portant modification des directives fixant les normes de base relatives à la protection sanitaire de la population et des travailleurs contre les dangers résultant des rayonnements ionisants. J. Off. Comm. Europ., 1980, L. 246.

[9] DOBICI F., FRITTELLI L., BELLI M., BIANCOTTO R., TACCONI A., NEGRI F. Experience in optimizing radiation protection in medical application of radiation. In : Radiation protection optimization "As Low As Reasonably Achievable", 2 nd European scientific seminar, Luxembourg, 8-9 Nov. 1983 (JOLIVET A., SINNAEVE J., Eds). EUR-9173. Luxembourg: Communautés européennes, 1984, 299-310.

[10] ERIXON O. Experience gained of dose reducing measures at Ringhals unit 2 during 1975 to 1983 . In : Radiation protection optimization "As Low As Reasonably Achievable", 2 nd European scientific seminar, Luxembourg, 8-9 Nov. 1983 (JOLIVET A., SINNAEVEJ., Eds). EUR-9173. Luxembourg: Communautés européennes, 1984, 139-151.

[11] FLEISHMAN A.B., NOTLEY H.M., WILKINSON J.M. Optimization of protection for gynecological radiotherapy. In": Radiation protection optimization "As Low As Reasonably Achievable", 2 nd European scientific seminar, Luxembourg, 8-9 Nov. 1983 (JOLIVET A., SINNAEVE J., Eds). EUR-9173. Luxembourg : Communautés européennes, 1984, 311-327.

[12] HEMMING C.R., KELLY G.N., CHARLES D. The influence of non-linear dose response relationship for late effects on the predict consequences of accidental releases of radioactive material. Rapport NRPB-M-91, 1983. 
[13] HOCK R., HECHT G. Minimization of occupational radiation exposure in KWU built nuclear power plants. In : Radiation protection optimization "As Low As Reasonably Achievable", 2 nd European scientific seminar, Luxembourg, 8-9 Nov. 1983 (JOLIVET A., SINNAEVE J., Eds) .EUR-9173. Luxembourg: Communautés européennes, $1984,169-200$.

[14] HOCK R. Minimization of radiation exposure of members of the public by effluents from nuclear power stations built by KWU. In : Radiation protection optimization "As Low As Reasonably Achievable", 2 nd European scientific seminar, Luxembourg, 8-9 Nov. 1983 (JOLIVET A., SINNAEVE J., Eds). EUR-9173. Luxembourg : Communautés européennes, 1984, 379-409.

[15] HUBERT P. The low-dose controversy and radiological risk assessment. Health Phys. 1983,45 (1) 144-149.

[16] HUBERT P., PAGES P. The application of ALARA principles to the safety of radioactive material transportation. In : Radiation protection optimization "As Low As Reasonably Achievable", 2 nd European scientific seminar, Luxembourg, 8-9 Nov. 1983 (JOLIVET A., SINNAEVE J., Eds). EUR-9173. Luxembourg : Communautés européennes, 1984, 451-478.

[17] HUNT G.J. Experience of ALARA in controlling radiocaesium discharges to the Irish sea. In : Radiation protection optimization "As Low As Reasonably Achievable", 2 nd European scientific seminar, Luxembourg, 8-9 Nov. 1983 (JOLIVET A., SINNAEVE J., Eds). EUR-9173. Luxembourg: Communautés européennes, 1984, 437-449.

[18] JEANSON P., LOCHARD J., MACCIA C., PAGES P. The analysis of dose reduction practices in the PWR units of Electricité de France. In : Radiation protection optimization "As Low As Reasonab!y Achievable", 2 nd European scientific seminar, Luxembourg, 8-9 Nov. 1983 (JOLIVET A., SINNAEVE J., Eds). EUR-9173. Luxembourg : Communautés européennes, 1984, 153-168.

[19] LOCHARD J., MACCIA C., PAGES P. L'évaluation de la protection radiologique des travailleurs dans les centrales nucléaires. Radioprotection, 1981, 16 (1) 201-208.

[20] LOCHARD J., MACCIA C., PAGES P. La valeur implicite de l'homme-sievert travailleur. Analyse de quelques mesures adoptées dans les réacteurs français à eau pressurisée. Radioprotection, 1982, 17 (2) 99-107.

[21] LOCHARD J. Optimization of radiation protection. Nucl. Safety, 1981, 22(4) 484-490.

[22] LOMBARD J., OUDIZ A., UZZAN G. La prise en compte du risque travailleur dans la mise en œuvre du principe d'optimisation des doses au public. Radioprotection, 1982, 17 (3) 155-162.

[23] NATIONAL ACADEMY OF SCIENCES. Report of the Advisory Committee on the biological effects of ionising radiations, BEIR III. Washington D.C. : National academy of sciences, 1980.

[24] OUDIZ A. Une approche multidimensionnelle de l'optimisation de la radioprotection. Radioprotection, 1980, 15 (1) 19-22.

[25] OUDIZ A., LOMBARD J., ZETTWOOG P. Un exemple d'optimisation de la protection radiologique des mineurs d'uranium. Radioprotection, 1984, 19 (1) 23-35.

[26] OUDIZ A., LOMBARD J., ZETTWOOG P. Optimization of short-lived radon daughters exposure in an underground uranium mine. In : Radiation protection optimization "As Low As Reasonably Achievable", 2 nd European scientific seminar, Luxembourg, 8-9 Nov. 1983. (JOLIVET A., SINNAEVE J., Eds). EUR-9173. Luxembourg : Communautés européennes, 1984, 235-250.

[27] PEPPER R.B., DUTTON L.M.C. The application of ALARA to the design and operation of the U.K. PWR. In : Radiation protection optimization "As Low As Reasonably Achievable", 2 nd European scientific seminar, Luxembourg, 8-9 Nov. 1983. (JOLIVET A., SINNAEVE J., Eds). EUR-9173. Luxembourg: Communautés européennes, $1984,263-297$. 
[28] RICHARDSON A.C.B., ROWE W.D. Optimization of mill tailings disposal practices. In : Radiation protection optimization "As Low As Reasonably Achievable", 2 nd European scientific seminar, Luxembourg, 8-9 Nov. 1983 (JOLIVET A., SINNAEVE J., Eds). EUR-9173. Luxembourg: Communautés européennes, 1984, 411-436.

[29] SPICKA M. Possibilities for reducing the radiation exposure in nuclear power plant with PWR through its design and during its operation. In : Radiation protection optimization "As Low As Reasonably Achievable", 2 nd European scientific seminar, Luxembourg, 8-9 Nov. 1983 (JOLIVET A., SINNAEVE J., Eds). EUR-9173. Luxembourg: Communautés européennes, 1984, 201-218.

[30] WAHLSTROEM B.G. Decontamination of steam generators at Loviisa nuclear power plant. The decision making and the results. In : Radiation protection optimization "As Low As Reasonably Achievable", 2 nd European scientific seminar, Luxembourg, 8-9 Nov. 1983. (JOLIVET A., SINNAEVE J., Eds). EUR-9173. Luxembourg: Communautés européennes, 1984, 219-234.

[31] ZETTWOOG P. Problèmes rencontrés dans la mise en œuvre du principe d'optimisation de la radioprotection. Radioprotection, 1983, 18 (3) 165-174. 